\title{
Enhancing SMM Image Steganography Method by using LSBraille Image Steganography Method (SMMWB; Secret Message Matching With Braille)
}

\author{
Abdelmgeid Amin Ali \\ Associate Professor, Dept. of Computer Science \\ Faculty of Science, Minia University, Egypt
}

\author{
Al - Hussien Seddik Saad \\ Assistant Lecturer, Dept. of Computer Science Faculty \\ of Science, Minia University, Egypt
}

\begin{abstract}
Information hiding techniques play a vital role in the recent years. Steganography is one of the important information hiding techniques which hides the existence of the message in the cover file. It gained importance in the past few years due to the increasing need for providing secrecy in an open environment like the internet. It can be defined as the art of hiding the fact that communication is taking place, by hiding information in other information. Many different carrier file formats can be used, but digital images are the most popular because of their frequency on the internet. Steganography is used to conceal the information so that no one can sense its existence. It has many technical challenges such as high hiding capacity and imperceptibility. The current technique proposed in this paper is based on previously proposed SMM image steganography method (Image Steganography By Matching Secret Message With Pixels of Cover Image or, Secret Message Matching) combined with previously proposed LSBraille image steganography method (Image Steganography Method By Using Braille Method of Blind People). The proposed method provides more MHC (Maximum Hiding Capacity) than the previously proposed method SMM combined with an excellent PSNR (Peak Signal to Noise Ratio).
\end{abstract}

\section{General Terms}

Image Steganography, Data Hiding In Digital Images.

\section{Keywords}

Spatial Domain Image Steganography, Peak Signal-to-Noise Ratio (PSNR), Maximum Hiding Capacity (MHC).

\section{INTRODUCTION}

Since the rise of the internet, one of the most important factors of information technology and Communication has been the security of information [1]. This is accomplished through data hiding. It is a method of hiding secret messages into a cover medium so that an unintended observer will not be aware of the existence of the hidden messages. Generally, messages will appear to be something else such as : images, articles, shopping lists, or some other cover text and, classically, the hidden message may be in invisible ink between the visible lines of a private letter [2]. This is achieved by steganography [3]. Steganography is the art and science of invisible communication which has received much attention from the scientific community recently [4]. The word steganography is derived from the Greek words "stegos" meaning "covered" and "grafia" meaning "writing" defining it as "covered writing" $[1,3]$.
Actually, there are three fundamental steganographic systems:

1. A pure steganographic system.

2. A secret key steganographic system.

3. A public key steganographic system.

In pure steganography, no keys is needed for the communicating parties other than the embedding and extracting algorithms. The security of such a system relies on the secrecy of the embedding and extracting algorithms [5]. While pure steganography does not involve the use or exchange of any secret information such as stego-keys, both secret key steganography and public key steganography rely on the sharing of such keys. In a secret key steganographic system, a sender hides a secret message into a cover object using a secret key. The key used in the embedding process can also be used to reverse the process in order to extract the hidden data. In this steganographic system, it is assumed that the communicating parties are able to transmit secret keys over a secure channel. Finally, public key steganography models after public key cryptography [5].

An important note is that, steganography is different from cryptography. The main objective of cryptography is to secure communications by changing the data into a form so that it cannot be understand by an eavesdropper. On the other hand, steganography techniques tend to hide the existence of the message itself, which makes it difficult for an observer to figure out where exactly the message is [6].

Steganography and cryptography are both ways to protect information from unwanted parties but neither technology alone is perfect and can be compromised. Once the presence of hidden information is revealed or even suspected, the purpose of steganography is partly defeated. The strength of steganography can thus be amplified by combining it with cryptography [1]. So, cryptography is used to supplement steganography, not replace it. If a hidden message is encrypted, it must also be decrypted if discovered, which provides another layer of protection [2].

Steganography is applied in various fields and applications like intelligence agencies, military agencies, medical imagery, TV broadcasting, advanced data structures, radar systems and remote sensing [7].

The rest of this paper is organized as follows. In section 2 , steganography basics, types and classification have been discussed. In section 3, image steganography, categories and performance are briefly explained. In section 4, the related works that the proposed method depends on are mentioned. In section 5, the proposed method is explained in details. In section 6 , the experimental results are given, discussed and 
summarized in tables and finally, section 7 concludes the paper.

\section{STEGANOGRAPHY}

\subsection{Basic Steganography system}

The Basic Steganography system is comprised of two algorithms, one for embedding and one for extracting. The embedding process is concerned with hiding a secret message within a cover work, and is the most carefully constructed process of the two. A great deal of attention is paid to ensure that the secret message goes unnoticed if a third party were to intercept the cover work. The extracting process is traditionally a much simpler process as it is simply an inverse of the embedding process, where the secret message is revealed at the end $[8,9]$. The entire process of steganography can be presented graphically as shown in Fig. 1.

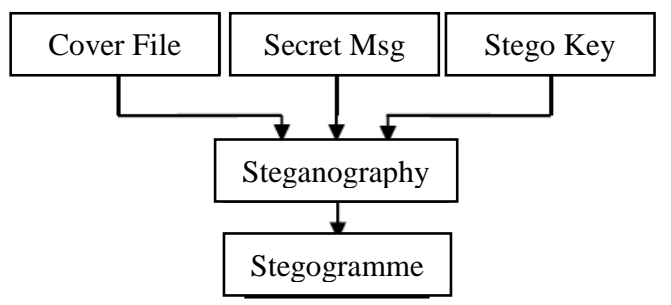

Fig. 1: Basic steganography system [10]

Fig. 1 shows an example of how steganography might be used in practice. In general, there are three inputs are required for the embedding process:-

- Cover file (Carrier): It is defined as the original file into which the required secret message is embedded. It is also termed as innocent file or host file [8].

- Payload (Secret Message): It is the secret massage that has to be embedded within the cover file. The payload can be in the form of text, audio, images, or video [8].

- Stegokey: is a password that may be used to encode the secret information to provide an additional level of security.

The next step is to pass these inputs through the stego-system encoder, which will be carefully engineered to embed the message within an exact copy of the cover work. The resulting output from the stego-system encoder is the stegogramme, which is designed to be as close to the cover work as possible, except it will contains the secret message.

- Stegogramme: is the final file obtained after embedding the payload into a given cover file. It should have similar properties to that of the cover file [8].

\subsection{Steganography Types \& Classification}

Actually, there are two basic types of steganography, linguistic and technical steganography.

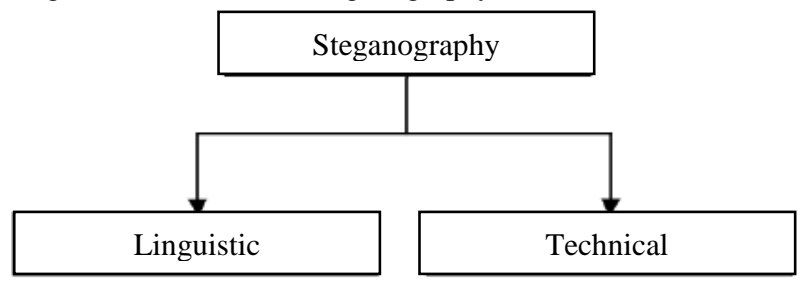

Fig. 2: Types of Steganography

\subsubsection{Linguistic Steganography}

Linguistic steganography is an art of concealing secret messages. More specifically, it takes advantage of the properties of natural language, such as the linguistic structure to hide messages. Linguistic steganography can be described quite simply as any form of steganography that uses language in the cover [11].

\subsubsection{Technical Steganography}

Technical steganography is a little broader in scope because it does not necessarily deal with the written word even though it communicates information. Technical steganography is the method of steganography where a tool, device, or method is used to conceal the message. In reality, linguistic steganography could be considered technical steganography because it is a method.

Technical steganography can be classified into: image, audio, video and text steganography [11]. As shown in Fig. 3, but we will concentrate only on image steganography as it is our point of research.

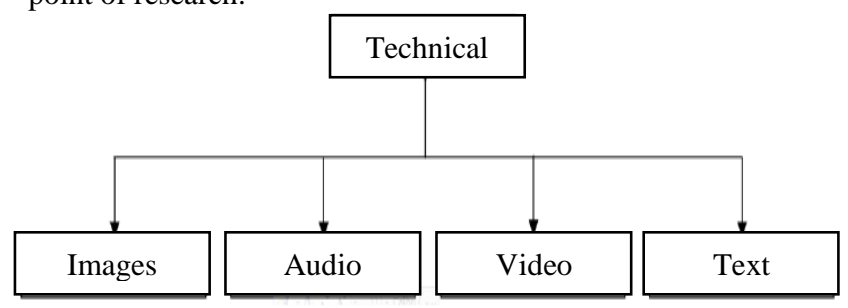

Fig. 3: Technical steganography classification

\section{IMAGE STEGANOGRAPHY}

\subsection{Digital Images}

As known, a computer image is an array of points called pixels which are represented as light intensity. The pixels are displayed horizontally row by row. In fact, Images are one of the most common types of cover objects used in modern digital steganography. These types of files are easy for any computer user to obtain or produce, and are prevalent enough in the world of computers that their presence alone does not warrant suspicion and that it would impractical to check every such file for hidden messages [11].

In particular, the presence of these types of files on the Internet - which is, by its far reaching and inherently anonymous nature, a common method for transmitting hidden communications - makes them a popular choice for steganographers [11].

\subsection{Image Steganography Categories}

The design of an image steganographic system can be categorized into spatial domain methods and transform domain methods.

In spatial domain methods, the processing is applied on the image pixel values directly. Such as, Least Significant Bit Insertion methods and Pallet based methods.

In transform domain methods, the first step is to transform the cover image into different domain. Then the transformed coefficients are processed to hide the secret information. These changed coefficients are transformed back into spatial domain to get stego image.

Steganography methods using DCT (Discrete Cosine Transforms), DWT (Discrete Wavelet Transforms), IWT, DFT (Discrete Fourier Transforms) come under this category.[12][13]

\subsection{Performance Of Image Steganography}

The performance of an image steganography system can be measured using several properties. The most important property is the undetectability (imperceptibility) of the data, 
which shows how difficult it is to determine the existence of a hidden message in the stego image and this can be measured by calculating the PSNR (Peak Signal to Noise Ratio). Other associated measure is the steganographic capacity or MHC (Maximum Hiding Capacity), which is the maximum information that can safely embedded in a work without having statistically detectable objects $[6,4]$.

\section{RELATED WORK}

In [10] authors proposed a new encoding technique that is called Mobile Phone Keypad encoding (MPK) for secret message that represent each character in the secret message by two digits only not three digits as ASCII encoding, and they constructed a full characters' table that contains small letters $(\mathrm{a} \ldots \mathrm{z})$, capital letters $(\mathrm{A} \ldots \mathrm{Z})$, digits $(0 \ldots$. 9) and special characters (@, -, +, ... ) and this proposed MPK encoding technique saved one third of the required space for embedding which in turn enhanced the Maximum Hiding Capacity (MHC) of the cover image, as a result of this the PSNR values have been enhanced too.

Also, in [14] the authors proposed a novel image steganography method based on Secret Message Matching (SMM). The proposed method works by adding an Alpha channel to the image whose pixels are 255 which means fully transparent channel, converting the R, G, B channels of the cover image into search spaces, converting the secret message into MPK digits [10], searching for these secret digits in the search space of the cover image and if found, the index is embedded in the corresponding alpha pixel by using Least Significant Bit (LSB) method otherwise, new search space will be encountered. The proposed method converts the whole cover image into a search space for the whole secret message. So, instead of embedding the secret character itself which can varies from (0 to 127); in case of secret text or (0 to 255); in case of secret image, only the secret character index which varies from (1 to 6) will be embedded; which are the indices of the search space. This means that the secret characters varies from $(001)_{2}$ to $(110)_{2}$ not from $(00000000)_{2}$ to $(11111111)_{2}$ which means smaller number of modified pixels (higher PSNR values) plus higher MHC.

Moreover, in [15] the authors proposed a method that hides the secret message inside the cover image by representing the secret message characters by using Braille method of reading and writing for blind people that can save more than onefourth of the required space for embedding. The proposed method is using the Braille method representations of the characters as each character is represented by only 6 dots using the $6-$ dots matrix which called (Braille Cell). The method will start by representing these characters (dots) as binary digits each of which consists of 6 bits only, not eight bits as in original LSB embedding method which uses the binary representation from the ASCII table. So, by using this representation one-fourth of the MHC for each cover image can be saved, which will increase the MHC and enhance the PSNR.

\section{PROPOSED METHOD}

After the previously proposed SMM image steganography method has been succeeded in PSNR and MHC compared to other methods, it has been found that there is a small probability that some digits of secret message may not be found in the whole search spaces of the cover image (worst case), because of this reason a try has been made to remove the probability of this worst case by combining the SMM method with LSBraille method in such a way that using the SMM method first, then ask if the whole message is embedded or not, if the whole message is not embedded, the LSBraille method will work by searching for the unchanged alpha pixels (Alpha $=255)$ and the method will use the search spaces corresponding to these Alpha pixels to embed the rest of secret digits in, and this proposed method has been called SMMWB (Secret Message Matching With Braille) method.

By applying this change to the SMM method, the SMMWB method will give us a higher embedding capacity than the original SMM and there won't be any loss in the pixels of cover image; they will be used all, and the maximum number of bytes that can be hidden in any image (MHC) can be calculated by using this formula:-

$$
\text { MHC }(\text { Bytes })=(\text { Image width } x \text { Image height }) / 2
$$

Table 1. Maximum Hiding Capacity of proposed method

\begin{tabular}{|c|c|c|}
\hline \multirow{2}{*}{ Image size (Pixels) } & \multicolumn{2}{|c|}{ MHC } \\
\cline { 2 - 3 } & Bytes & bits \\
\hline $8 \times 8$ & 32 & 256 \\
\hline $16 \times 16$ & 128 & 1,024 \\
\hline $32 \times 32$ & 512 & 4,096 \\
\hline $64 \times 64$ & 2,048 & 16,384 \\
\hline $128 \times 128$ & 8,192 & 65,536 \\
\hline $256 \times 256$ & 32,768 & 262,144 \\
\hline $512 \times 512$ & 131,072 & $1,048,576$ \\
\hline $1024 \times 1024$ & 524,288 & $4,194,304$ \\
\hline
\end{tabular}

As shown in table 1, after this modification, the SMMWB method will has a very high embedding capacity without any probability of pixels loss as the previous SMM method.

The proposed method will work as in the following algorithm:-

Algorithm: Message Embedding Using SMMWB Method

Input: Cover Image C; Secret Message M.

Output: StegoImage S

Steps

1) Construct Alpha channel same Width and Height of C.

2) Make all the pixels of Alpha equal to 255

3) Split the image into Red (R), Green (G), Blue (B).

4) Convert $R, G$ and $B$ into vectors;

$$
\begin{aligned}
& \mathrm{R}=\left\{\mathrm{r}_{1}, \mathrm{r}_{2}, \ldots, \mathrm{r}_{\mathrm{WxH}}\right\} \\
& \mathrm{G}=\left\{\mathrm{g}_{1}, \mathrm{~g}_{2}, \ldots ., \mathrm{g}_{\mathrm{WxH}}\right\} \\
& \mathrm{B}=\left\{\mathrm{b}_{1}, \mathrm{~b}_{2}, \ldots ., \mathrm{b}_{\mathrm{WxH}}\right\}
\end{aligned}
$$

5) Divide the three vectors into blocks each of which contains 3 pixels $\left(\mathrm{r}_{\mathrm{i}}, \mathrm{g}_{\mathrm{i}}, \mathrm{b}_{\mathrm{i}}\right) ;(1 \leq \mathrm{i} \leq \mathrm{WxH})$

6) Construct search spaces by taking $2 \mathrm{nd}$ and 3rd digits from each pixel; $\mathrm{SS}_{\mathrm{i}}=\left\{\mathrm{r}_{\mathrm{i}}(2), \mathrm{r}_{\mathrm{i}}(3), \mathrm{g}_{\mathrm{i}}(2), \mathrm{g}_{\mathrm{i}}(3), \mathrm{b}_{\mathrm{i}}(2), \mathrm{b}_{\mathrm{i}}(3)\right\}$

7) Convert Alpha into a vector; $A=\left\{a_{1}, a_{2}, \ldots ., a_{W x H}\right\}$

8) Encode secret message M using MPK Encoding; MPKDigits $=\left\{\mathrm{md}_{1}, \mathrm{md}_{2}, \ldots \ldots, \mathrm{md}_{\mathrm{n}}\right\}$

9) $\mathrm{i}=1, \mathrm{k}=1$

10) If $\left(\mathrm{md}_{\mathrm{i}}\right.$ Match $\left.\mathrm{SS}_{\mathrm{k}}\right)$

Pos $=$ index 


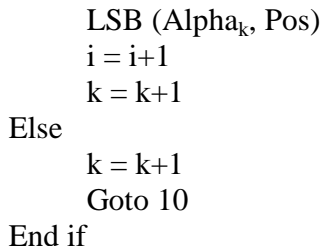

11) Repeat step 10 until the whole MPKDigits have been matched and their indices are embedded or search spaces have been completed.

12) if (not the whole digits are embedded)

Search for empty Alpha pixels.

- $\quad$ Embed the rest of digits in the search spaces corresponding to empty Alpha pixels using LSBraille.

12) Convert A, R, G and B back into channels.

13) Merge the four channels A, R, G and B respectively to construct the ARGB StegoImage $S$.

Now, after the proposed method has been discussed in details, an evaluation for the performance of SMMWB proposed method will be made by taking the same messages and embed them in the same cover images that have been used in earlier methods.

\section{EXPERIME NTAL RESULTS}

In this section, the proposed method has been tested by taking different messages with different lengths and hiding them in some cover images. The results that are obtained from these experiments are recorded and can be summarized in the following tables:-

Table 2. PSNR of proposed method after embedding MHC that a cover image can hold

\begin{tabular}{|c|c|c|}
\hline \multirow[b]{2}{*}{ Cover images } & \multirow{2}{*}{$\begin{array}{c}\text { Message } \\
\text { Capacity } \\
\text { (Full Capacity) }\end{array}$} & PSNR (dB) \\
\hline & & $\begin{array}{c}\text { Proposed } \\
\text { Method }\end{array}$ \\
\hline Lena $128 \times 128$ & \multirow{3}{*}{$\begin{array}{c}8,192 \text { Bytes } \\
= \\
65,536 \text { Bits }\end{array}$} & 45.287281 \\
\hline Baboon $128 \times 128$ & & 44.763127 \\
\hline Peppers $128 \times 128$ & & 45.190245 \\
\hline Lena $256 \times 256$ & \multirow{3}{*}{$\begin{array}{c}32,768 \text { Bytes } \\
= \\
262,144 \text { Bits }\end{array}$} & 45.332465 \\
\hline Baboon $256 \times 256$ & & 44.853816 \\
\hline Peppers $256 \times 256$ & & 45.372828 \\
\hline Lena 512 × 512 & \multirow{3}{*}{$\begin{array}{c}131,072 \text { Bytes } \\
= \\
1,048,576 \text { Bits }\end{array}$} & 45.499052 \\
\hline Baboon $512 \times 512$ & & 45.143743 \\
\hline Peppers $512 \times 512$ & & 45.736227 \\
\hline
\end{tabular}

In table 2, the performance of the proposed SMMWB method has been evaluated by embedding maximum number of bytes that a cover image can hold in different cover images with different sizes and the results showed that the PSNR values were very high and the cover and stego images look identical.

Table 3. Comparison between PSNRs of method in [16] and the proposed method

\begin{tabular}{|c|c|c|c|}
\hline \multirow{2}{*}{$\begin{array}{c}\text { Cover } \\
\text { images }\end{array}$} & \multirow{2}{*}{$\begin{array}{c}\text { Message } \\
\text { Capacity }\end{array}$} & \multicolumn{2}{|c|}{ PSNR (dB) } \\
\cline { 3 - 4 } (512 x 512) & (Bits) & {$[\mathbf{1 6}]$} & $\begin{array}{c}\text { Proposed } \\
\text { Method }\end{array}$ \\
\hline Lena & 145,787 & 42.26 & 50.622882 \\
\hline Baboon & 144,916 & 38.44 & 50.456067 \\
\hline Pepper & 145,995 & 42.28 & 50.754112 \\
\hline
\end{tabular}

Table 3 compared the proposed SMMWB method with " color image steganography based on pixel value differencing in spatial domain" method [16] by hiding (145,787 - 144,916 145,995) secret bits in 512 x 512 cover images (Lena, Baboon, Pepper) respectively, and it has been found that the proposed method has more PSNR values than method in [16] which means that, the stego image quality of SMMWB method will be higher than the stego image quality of this method.

Table 4. Comparison between PSNRs of method in [13] and the proposed method

\begin{tabular}{|c|c|c|c|}
\hline \multirow{2}{*}{$\begin{array}{c}\text { Cover } \\
\text { images }\end{array}$} & \multirow{2}{*}{$\begin{array}{c}\text { Message } \\
\text { Capacity } \\
\text { (256 x 256) }\end{array}$} & \multicolumn{2}{|c|}{ PSNR (dB) } \\
\cline { 3 - 4 } (Bytes) & {$[\mathbf{1 3}]$} & $\begin{array}{c}\text { Proposed } \\
\text { Method }\end{array}$ \\
\hline Baboon & 16,384 & 44.8 & 45.254335 \\
\hline Pepper & 16,384 & 44.7 & 45.824773 \\
\hline
\end{tabular}

Also, table 4 compared the proposed method with "a secure and high capacity image steganography" method [13] by hiding $(16,384)$ secret characters (Bytes) in 256 x 256 cover images (Baboon, Pepper), and it has been found that the proposed method has more PSNR values than method in [13] which means that higher stego image quality.

Table 5. Comparison between PSNRs of method in [17] and the proposed method

\begin{tabular}{|c|c|c|c|}
\hline \multirow{2}{*}{$\begin{array}{c}\text { Cover } \\
\text { images }\end{array}$} & \multirow{2}{*}{$\begin{array}{c}\text { Message } \\
\text { Capacity }\end{array}$} & \multicolumn{2}{|c|}{ PSNR (dB) } \\
\cline { 3 - 4 } (512 x 512) & (Bits) & {$[\mathbf{1 7 ]}$} & $\begin{array}{c}\text { Proposed } \\
\text { Method }\end{array}$ \\
\hline Lena & 4,096 & 42.9 & 66.461596 \\
\hline Lena & 10,000 & 38.38 & 62.501075 \\
\hline Pepper & 4,096 & 41.87 & 66.025166 \\
\hline Pepper & 10,000 & 37.78 & 62.291950 \\
\hline
\end{tabular}

Moreover, table 5 compared the proposed method with "secure information transmission using steganography and morphological associative memory" method [17] by hiding $(4,096-10,000-4,096-10,000)$ secret bits in $512 \times 512$ cover images (Lena, Pepper) respectively, and also, it has been found that the proposed method has more PSNR values than method [17].

Table 6. Comparison between PSNRs of $1^{\text {st }}$ method in [18] and the proposed method

\begin{tabular}{|c|c|c|c|}
\hline \multirow{2}{*}{$\begin{array}{c}\text { Cover } \\
\text { images }\end{array}$} & \multirow{2}{*}{$\begin{array}{c}\text { Message } \\
\text { Capacity }\end{array}$} & \multicolumn{2}{|c|}{ PSNR (dB) } \\
\cline { 3 - 4 }$(\mathbf{5 1 2}$ x 512) & (Bits) & $\begin{array}{c}\text { Four } \\
\text { Neighbors }\end{array}$ & $\begin{array}{c}\text { Proposed } \\
\text { Method }\end{array}$ \\
\hline Lena & 392,208 & 41.1468 & 46.281893 \\
\hline baboon & 435,223 & 36.5154 & 45.705512 \\
\hline Pepper & 393,567 & 41.0449 & 46.359389 \\
\hline
\end{tabular}

In table 6 a comparison between proposed SMMWB method and "four neighbors' " method [18] by hiding $(392,208$ 435,223 - 393,567) secret bits in 512 x 512 cover images (Lena, Baboon, Pepper) respectively, and also SMMWB method succeeded in obtaining higher PSNR values.

Table 7. Comparison between PSNRs of $2^{\text {nd }}$ method in [18] and the proposed method

\begin{tabular}{|c|c|c|c|}
\hline \multirow{2}{*}{$\begin{array}{c}\text { Cover } \\
\text { images } \\
(\mathbf{5 1 2} \text { x 512) }\end{array}$} & $\begin{array}{c}\text { Message } \\
\text { Capacity } \\
\text { (Bits) }\end{array}$ & $\begin{array}{c}\text { Eight } \\
\text { Neighbors }\end{array}$ & $\begin{array}{c}\text { Proposed } \\
\text { Method }\end{array}$ \\
\hline Lena & 395,680 & 40.6504 & 49.306142 \\
\hline baboon & 443,165 & 35.0725 & 48.617474 \\
\hline Pepper & 395,213 & 40.5709 & 49.353188 \\
\hline
\end{tabular}


Another comparison has been made in table 7, between proposed method and "eight neighbors' " method [18] by hiding $(395,680$ - 443,165 - 395,213) secret bits in 512 x 512 cover images (Lena, Baboon, Pepper) respectively, and proposed method's PSNR values were also higher.

Table 8. Comparison between PSNRs of $3^{\text {rd }}$ method in [18] and the proposed method

\begin{tabular}{|c|c|c|c|}
\hline \multirow{2}{*}{$\begin{array}{c}\text { Cover } \\
\text { images }\end{array}$} & \multirow{2}{*}{$\begin{array}{c}\text { Message } \\
\text { Capacity }\end{array}$} & \multicolumn{2}{|c|}{ PSNR (dB) } \\
\cline { 3 - 4 }$(\mathbf{5 1 2}$ x 512) & (Bits) & $\begin{array}{c}\text { Diagonal } \\
\text { Neighbors }\end{array}$ & $\begin{array}{c}\text { Proposed } \\
\text { Method }\end{array}$ \\
\hline Lena & 196,968 & 43.9590 & 46.244887 \\
\hline Baboon & 220,575 & 38.8280 & 45.643654 \\
\hline Pepper & 197,379 & 43.7135 & 46.357712 \\
\hline
\end{tabular}

Also, here in table 8 another comparison has been made between SMMWB method and "diagonal neighbors' " method [18] by hiding (196,968 - 220,575 - 197379) secret bits in 512 x 512 cover images (Lena, Baboon, Pepper) respectively, and it has been found that the proposed method has more PSNR values than diagonal neighbors' method [18] which means that, the stego image quality of SMMWB method will be higher than the stego image quality of this method.

Table 9. Comparison between PSNRs of $1^{\text {st }}$ method in [19] and the proposed method

\begin{tabular}{|c|c|c|c|}
\hline \multirow{2}{*}{$\begin{array}{c}\text { Cover } \\
\text { images }\end{array}$} & \multirow{2}{*}{$\begin{array}{c}\text { Message } \\
\text { Capacity } \\
\text { (512 x 512) }\end{array}$} & \multicolumn{2}{|c|}{ PSNR (dB) } \\
\cline { 3 - 4 } (Bits) & $\begin{array}{c}\text { MSM } \\
\text { 2 Sided }\end{array}$ & $\begin{array}{c}\text { Proposed } \\
\text { Method }\end{array}$ \\
\hline Baboon & 695,145 & 34.14 & 45.428993 \\
\hline Lena & 397,513 & 41.85 & 46.225442 \\
\hline Airplane & 342,185 & 41.58 & 47.121592 \\
\hline
\end{tabular}

Moreover, in table 9 the comparison results showed that SMMWB 's PSNR values were higher than PSNR values of "the modified side match scheme for image steganography; two sided case" method [19] after embedding (695,145 397,513 - 342,185) secret bits in 512 x 512 cover images (Baboon, Lena, Airplane) respectively.

Table 10. Comparison between PSNRs of $2^{\text {nd }}$ method in [19] and the proposed method

\begin{tabular}{|c|c|c|c|}
\hline \multirow{2}{*}{$\begin{array}{c}\text { Cover } \\
\text { images }\end{array}$} & \multirow{2}{*}{$\begin{array}{c}\text { Message } \\
\text { Capacity } \\
\text { (512 x 512) }\end{array}$} & \multicolumn{2}{|c|}{ PSNR (dB) } \\
\cline { 3 - 4 } (Bits) & $\begin{array}{c}\text { MSM } \\
\text { 3 Sided }\end{array}$ & $\begin{array}{c}\text { Proposed } \\
\text { Method }\end{array}$ \\
\hline Baboon & 498,167 & 35.24 & 45.595915 \\
\hline Lena & 274,548 & 45.52 & 47.799213 \\
\hline Airplane & 239,472 & 45.06 & 48.628654 \\
\hline
\end{tabular}

In table 10, a comparison between SMMWB method and "three sided case" method [19] has been done by hiding $(498,167-274,548$ - 239,472) secret bits in 512 x 512 cover images (Baboon, Lena, Airplane) respectively, and also SMMWB was succeeded in the comparison.

Table 11. Comparison between PSNRs of $3^{\text {rd }}$ method in [19] and the proposed method

\begin{tabular}{|c|c|c|c|}
\hline \multirow{2}{*}{$\begin{array}{c}\text { Cover } \\
\text { images } \\
(512 \times 512)\end{array}$} & \multirow{2}{*}{$\begin{array}{c}\text { Message } \\
\text { Capacity } \\
\text { (Bits) }\end{array}$} & \multicolumn{2}{|c|}{ PSNR (dB) } \\
\hline & & $\begin{array}{c}\text { MSM } \\
4 \text { Sided }\end{array}$ & $\begin{array}{c}\text { Proposed } \\
\text { Method }\end{array}$ \\
\hline Baboon & 306,209 & 39.31 & 47.207721 \\
\hline Lena & 168,289 & 48.64 & 50.010883 \\
\hline Airplane & 151,843 & 48.23 & 50.607618 \\
\hline
\end{tabular}

Again in table 11 the proposed method succeeded in the PSNR comparison with "four sided case" method [19] by hiding $(306,209$ - 168,289 - 151,843) secret bits in 512 x 512 cover images (Baboon, Lena, Airplane) respectively.

Table 12. Comparison between PSNRs of method in [11] and the proposed method

\begin{tabular}{|c|c|c|c|}
\hline \multirow{2}{*}{$\begin{array}{c}\text { Cover images } \\
(\mathbf{5 1 2} \times \mathbf{5 1 2})\end{array}$} & $\begin{array}{c}\text { Message } \\
\text { Capacity } \\
(\text { Bits) }\end{array}$ & \multicolumn{2}{|c|}{ PSNR (dB) } \\
\cline { 3 - 4 } & 606,688 & 45.72295 & $\begin{array}{c}\text { Proposed } \\
\text { Method }\end{array}$ \\
\hline Lena & 65.892206 \\
\hline Baboon & 659,256 & 45.33670 & 45.458835 \\
\hline Peppers & 604,632 & 45.42819 & 46.149193 \\
\hline
\end{tabular}

Finally, table 12 compared the SMMWB proposed method with "ESLDIP" method [11] by hiding (606,688 - 659,256 604,632) secret bits in 512 x 512 cover images (Lena, Baboon, Peppers) respectively and the proposed method succeeded in the comparison results.

\section{CONCLUSION}

In this paper, an enhancement has been made to the previously proposed image steganography method SMM (secret message matching) by combining the LSBraille image steganography method with it.

The proposed method was compared with a lot of methods as shown from table 2 to table 12, it has been found that the proposed SMMWB method has more PSNR values than other methods which means the stego image quality of the proposed method will be higher than the quality of other methods.

This means that this enhancement has been succeeded to improve the maximum hiding capacity (MHC) as stated in table 1 while keeping the PSNR values higher than other methods.

Finally, after checking the results, it can be said that the proposed enhancement made the SMMWB method more efficient than the original SMM and other image steganography methods.

\section{FUTURE WORK}

By reaching to this point in our researches, we can say that we were succeeded to propose a very efficient image steganography method with a very high MHC and a wonderful PSNR values as shown in the experimental results section.

As a future work, we will try to enhance the security of the proposed SMMWB method to make it a fully efficient image steganography system which keeping the data inside it secured, even if the data has been extracted.

\section{REFERENCES}

[1] Kanzariya N. K., Nimavat A. V., " Comparison of Various Images Steganography Techniques ", International Journal of Computer Science and Management Research, Vol 2, Issue 1, January 2013.

[2] Sravanthi G. S., Sunitha B. D., Riyazoddin S.M., Janga M. R., " A Spatial Domain Image Steganography Technique Based on Plane Bit Substitution Method ", Global Journal of Computer Science and Technology, Graphics \& Vision, Vol 12, Issue 15, Version 1.0, 2012.

[3] Poornima R., Iswarya R.J., " An Ooverview Of Digital Image Steganography ", International Journal of Computer Science \& Engineering Survey (IJCSES), Vol 4, No.1, February 2013.

[4] Sushil K., Muttoo S. K., " A Comparative Study Of 
Image Steganography In Wavelet Dmain ", International Journal of Computer Science and Mobile Computing (IJCSMC), Vol. 2, Issue. 2, February 2013.

[5] Chinchu E. A., Iwin T. J., " An Analysis Of Various Steganographic Algorithms ", International Journal of Advanced Research in Electronics and Communication Engineering (IJARECE), Vol 2, Issue 2, February 2013.

[6] Shikha S., Sumit B., " Image Steganography: A Review ", International Journal of Emerging Technology and Advanced Engineering, Vol 3, Issue 1, January 2013.

[7] Vanmathi C., Prabu S., " A Survey of State of the Art techniques of Steganography ", International Journal of Engineering and Technology (IJET), Vol 5, No 1, FebMar 2013.

[8] Abdelmgeid A. A., Al - Hussien S. S., " New Text Steganography Technique by using Mixed-Case Font ", International Journal of Computer Applications, Vol 62, No.3, January 2013.

[9] Deepa S., Umarani R., " A Study on Digital Image Steganography ", International Journal of Advanced Research in Computer Science and Software Engineering, Vol 3, Issue 1, January - 2013.

[10] Abdelmgeid A. A., Al - Hussien S. S., " New Technique for Encoding the Secret Message to Enhance the Performance of MSLDIP Image Steganography Method (MPK Encoding) ", International Journal of Computer Applications, Vol 59, No.15, December 2012.

[11] Al-Hussien S. S., " Enhancing the (MSLDIP) Image steganographic method (ESLDIP Method) ", International Conference on Graphic and Image Processing (ICGIP 2011), Proc. of SPIE Vol. 8285, Issue 82853I, 2011.
[12] Hemalatha S., Dinesh A., Renuka A., Priya R. K., " A Secure And High Capacity Image Steganography Technique ", An International Journal (SIPIJ), Vol.4, No.1, February 2013

[13] Sneha A., Sanyam A., " A Proposed Method for Image Steganography Using Edge Detection ", International Journal of Emerging Technology and Advanced Engineering, Vol 3, Issue 2, February 2013.

[14] Abdelmgeid A. A., Al - Hussien S. S., " New Image Steganography Method By Matching Secret Message With Pixels Of Cover Image (SMM) ", International Journal of Computer Science Engineering and Information Technology Research (IJCSEITR), Vol. 3, Issue 2, Jun 2013.

[15] Abdelmgeid A. A., Al - Hussien S. S., " Image Steganography Technique By Using Braille Method of Blind People (LSBraille) ", International Journal of Image Processing (IJIP), Vol 7, Issue 1, 2013.

[16] Mandal J. K., Debashis D., " Colour Image Steganography Based on Pixel Value Differencing in Spatial Domain ", International Journal of Information Sciences and Techniques (IJIST), Vol. 2, No. 4, July 2012.

[17] Sara N., Amir M. E., Mohammad S. M., " Secure Information Transmission using Steganography and Morphological Associative Memory ", International Journal of Computer Applications, Vol 61, No 7, January 2013.

[18] Moazzam H., Sadia A. H., Farhana S., " Variable Rate Steganography in Gray Scale Digital Images Using Neighborhood Pixel Information ", The International Arab Journal of Information Technology, Vol. 7, No. 1, January 2010

[19] Po-Yueh C., Wei-En W., " A Modified Side Match Scheme for Image Steganography ", International Journal of Applied Science and Engineering, Vol 7, No 1,2009 